\title{
Ein unvollständiges und unausgewogenes Bild
}

\section{Thomas Cueni}

Generalsekretär Interpharma

Der Autor schlägt in seinem Beitrag einen weiten Bogen von der Deontologie über Treu und Glauben, öffentliches Interesse, Verfehlungen der Pharmaindustrie, Versäumnisse von Ärzten, vor allem von Meinungsbildnern, Machenschaften der Tabakindustrie, Druck der Wirtschaftslobby, Versagen von Politikern usw. Angesichts der Breite der Themen entsteht notgedrungen ein unvollständiges und leider unausgewogenes Bild, bei dem einzelne Aspekte emotional und fragmentarisch überzeichnet werden.

Wir beschränken uns im Folgenden auf die Auseinandersetzung mit Fragen, welche die Pharmaindustrie betreffen.

Ausgangs- und Mittelpunkt der Überlegungen des Autors bildet das Vertrauen als Grundlage des menschlichen Zusammenlebens. Dies gelte auch für die auf den ersten Blick bilaterale Beziehung zwischen Arzt und Patient, die aber durch das "öffentliche Interesse» verkompliziert werde, dem der Arzt als Angestellter des Spitals und als Kostenverursacher bei Versicherungen und beim Staat ausgesetzt sei. Nach dieser Einleitung wendet sich der Autor «der ärztlichen Expertise im Medikamentenmarkt und in der öffentlichen Gesundheit» zu und befasst sich mit dem Fall des japanischen Kardiologen Matsubara.

Nach Datenmanipulationen bei klinischen Studien war dieser 2013 von seiner Stelle an der Universität Kyoto zurückgetreten. In der Folge wurden verschiedene seiner Publikationen aus namenhaften Journals zurückgezogen sowie Untersuchungen und Verfahren eingeleitet. Der Autor unterstellt eine Instrumentalisierung der Ärzteschaft und weist darauf hin, dass neben dem betroffenen Forscher das öffentliche Interesse zu beachten sei, weil das Vertrauen in akademische Institutionen und Spitäler, in deren Mitarbeiter und Experten beschädigt worden sei.

Im folgenden Abschnitt geht der Autor auf eine $\mathrm{Pu}$ blikation von Peter C. Gøtzsche [1, 2] ein, der zehn Fälle von grossen Pharmafirmen aus den USA beschreibt, die im Rahmen von Vergleichen mit Behörden oder wegen Verurteilungen beträchtliche Bussen bezahlen mussten. Es handelt sich bei den Vergehen zum grössten Teil um Bewerbung nicht zugelassener Indikationen (Off-Label-Use), ferner falsche Darstellung von Forschungsresultaten, Verheimlichung von Risiken sowie Betrug zu Lasten von Medicaid und Medicare.

Die Kritik des Autors an den beschriebenen Verhaltensweisen ist durchaus berechtigt. Die Vergehen sind behördlich untersucht und mit Verurteilungen oder Vergleichen abgeschlossen worden. In einem Fall ist das Verfahren noch hängig. Die Verstösse betreffen aber nicht nur die direkt Betroffenen oder das «öffentliche Interesse», sondern sie schaden auch der Pharmaindustrie. Diese ist auf das Vertrauen und eine gute Zusammenarbeit mit Ärzten, Meinungsbildnern, Behörden und Patienten angewiesen. Für die Entwicklung von Medikamenten und für die sachgerechte wissenschaftliche Information braucht sie die Unterstützung medizinischer Experten. Auch gemäss den Richtlinien der Schweizerischen Akademie der medizinischen Wissenschaften liegt die Zusammenarbeit

\section{Die Verstösse treffen nicht nur die direkt} Betroffenen oder das "öffentliche Interesse", sie schaden auch der Pharmaindustrie.

von Ärzten mit der Industrie im Interesse einer guten Gesundheitsversorgung und der Wissenschaft [3]. Dass Firmen systematisch, wie im Artikel dargestellt, gesetzeswidrige Praktiken als Marketingmassnahmen zur Profitoptimierung einplanen, widerspricht einer rationalen, wirtschaftlichen und langfristig ausgerichteten Unternehmensführung. Dieser Vorwurf ist deshalb ebenso unzutreffend wie diffamierend.

Gerade der häufigste Verstoss, die Bewerbung von Off-Label-Indikationen, eignet sich nicht als «Marketingmassnahme», da ein Vergleich von Werbeaussagen mit der offiziellen Fachinformation das Vergehen sofort offensichtlich macht. Jede Fachperson kann diesen Vergleich problemlos durchführen und Pharmafirmen sind diesbezüglich aufmerksam und verzeigen Mitbewerber bei vermuteten oder offensichtlichen Verstössen. Dass verantwortliche Manager Off-Label- 
Kampagnen planen, ist kaum vorstellbar, weil sie sich selber und dem Unternehmen schaden würden. Aus individuellen Unzulänglichkeiten auf ein systemisch falsches Verhalten zu schliessen, ist daher unstatthaft. Da Grossunternehmen, wie sie viele Pharmafirmen darstellen, zahlreiche Interessen und Ziele wie Profitabilität, Innovation, Sicherheit usw. verfolgen, sind organisationsinterne Zielkonflikte kaum zu vermeiden. Um diese vor allem in sensiblen Bereichen zu lösen, gelten für Mitarbeitende der Firmen entsprechende Verhaltensregeln. Für die Beziehungen zur Ärzteschaft hat die Pharmaindustrie branchenweite Vereinbarungen getroffen, zu denen sich alle grösseren Pharmaunternehmen verpflichtet haben. In der Schweiz sind dies der Pharma-Kodex [4] und der Pharma-Kooperations-Kodex [5] sowie international der IFPMA Code of Practice 2012 [6]. Diese Verhaltensregeln der Pharmaindustrie sind veröffentlicht; sie sind Ärzten, Spitälern, Fach- und Standesorganisationen, Behörden, Politikern und andern Betroffenen bekannt. Sie sind auch für jeden Teil der «interessierten Öffentlichkeit» jederzeit einsehbar.

Die Umsetzung von unternehmensweiten Verhaltensregeln für Mitarbeitende ist besonders in grossen Organisationen eine anspruchsvolle Managementaufgabe. Die Pharmaunternehmen haben hier beträchtliche Anstrengungen unternommen: Ausbau von Compliance-Abteilungen, Vermehrung und Verfeinerung von Vorschriften, regelmässige Schulung von Mitarbeitenden, interne Kontrollen von allen Werbeund Informationsmaterialien, organisatorische Trennung von Marketing- und Medizinabteilungen mit Entflechtung ihrer Aufgaben, Beschränkung der Zuständigkeit von Marketing- und Verkaufsabteilungen auf zugelassene Medikamente und Indikationen.

Man darf auch annehmen, dass die Mitarbeitenden der Pharmaindustrie so gesetzestreu wie andere Personen sind. Zudem sind sie nicht nur Firmenvertreter, sondern auch Bürger, Familienangehörige, Freunde und Patienten. Auch ihnen ist als normale Mitglieder der Gesellschaft das «öffentliche Interesse» ein wichtiges Anliegen und sie nehmen ihre Verantwortung wahr, wie in einigen der erwähnten Fälle dokumentiert, so dass dank «Whistleblowern» Verstösse ans Licht kommen.

In seinem Panorama der Kritik findet der Autor immerhin eine Ausnahme für ein gutes Vorgehen im Gesundheitswesen: die Bekämpfung der AIDS-Epidemie. In der Tat konnte hier, da alle wichtigen gesellschaftlichen Kräfte wie Ärzte, Gesundheitsbehörden, Politik usw. die gleichen Interessen verfolgten, rasch viel erreicht werden. Dass die Pharmaindustrie durch die Entwicklung von Tests und wirksamen Medikamenten hierbei einen wichtigen Beitrag geleistet hat, findet der Autor leider nicht der Erwähnung wert.

\section{Aus individuellen Unzulänglichkeiten auf ein systemisch falsches Verhalten zu schliessen, ist unstatthaft.}

Der Interpharma als Verband der forschenden pharmazeutischen Firmen der Schweiz sowie allen ihren Mitgliedsfirmen ist ein Geschäftsgebaren, das im Einklang mit den gesetzlichen Anforderungen sowie hohen ethischen Standards steht, ein fundamentales Anliegen. Sie verwahrt sich gegen die im Artikel erhobenen pauschalen, undifferenzierten und diffamierenden Anschuldigungen; ist aber immer zum konstruktiven Gespräch bereit und bietet Hand für weitere mögliche Verbesserungen in den Beziehungen zwischen der Ärzteschaft und der Pharmaindustrie zum anhaltenden Nutzen der Patientinnen und Patienten.

\section{Referenzen:}

1 Gøtzsche PC. Big pharma often commits corporate crime, and this must be stopped. BMJ. 2012;345:e8462.

2 G $\varnothing$ tzsche PC. Corporate crime in the pharmaceutical industry in common, serious and repetitive. http://logueos.com/ adjuntos/1379506341-Corporate-crime-long-version.pdf

3 Schweizerische Akademie der Medizinischen Wissenschaften, Richtlinien Zusammenarbeit Ärzteschaft - Industrie, Version 13.

4 Verhaltenskodex der pharmazeutischen Industrie in der Schweiz (Pharmakodex), www.scienceindustries.ch

5 Verhaltenskodex der pharmazeutischen Industrie in der Schweiz über die Zusammenarbeit mit Fachkreisen und Patientenorganisationen (Pharma-Kooperations-Kodex), Stand November 2014, www.scienceindustries.ch

6 IFPMA Code of Practice 2012. www.ifpma.org/ethics/ ifpma-code-of-practice/about-ifpma-code-of-practice.html 